\title{
Amount Recovered Infinity Predicted
}

National Cancer Institute

\section{Source}

National Cancer Institute. Amount Recovered Infinity Predicted. NCI Thesaurus. Code

C112033.

The cumulative amount recovered extrapolated to infinity, calculated using the predicted value of the last non-zero concentration. 